\title{
The Application of VaR Model in Interest Rate Risk Management of Commercial Banks in China
}

\author{
Jianhua Zhu Tiandi Liu \\ Guizhou University of Finance and Economics
}

\begin{abstract}
With the reform of interest rate liberalization in China, the main risks of commercial Banks in China are gradually turned into interest rate risks. However, at present, China's commercial Banks are not aware of the risk of interest rate, avoid problems such as the lack of interest rate risk tools, and there are many risks such as repricing risk and base difference risk. Controlling interest rate risk becomes the main content of risk management of commercial Banks. This article selects 20102016 Shanghai overnight interest rates in the interbank market to simulate variable market interest rate, our country commercial bank interest rate risk value for empirical research, and to our country commercial bank interest rate risk management puts forward the corresponding Suggestions.
\end{abstract}

\section{Keywords}

Commercial Banks; Interest rate risk management; The VaR model

\section{VaR 模型在我国商业银行利率风险管理 中的应用分析}

\author{
朱建华 刘甜娣 \\ 贵州财经大学, 贵州贵阳 550025, 中国
}

\begin{abstract}
摘要: 随着我国利率市场化改革不断推进, 我国商业银行主要风险逐渐转变为利率风险。但 目前我国商业银行对于利率风险存在意识浅薄, 规避和管理利率风险工具贵乏等问题, 还存 在着诸多重新定价风险、基差风险等隐患。管控利率风险成为商业银行风险管理主要内容。 本文选取 2010-2016 年上海同业拆借市场中的隔夜拆借利率为模拟市场利率变量, 对我国 商业银行的利率风险价值进行实证研究, 并对我国商业银行利率风险管理提出了相应的建 议。
\end{abstract}

关键词: 商业银行 ; 利率风险管理 ; VaR 模型 


\section{1.引言}

巴塞尔银行监督委员会在《利率 风险管理与监督原则》中将利率风险 定义为利率变化使商业银行的实际收 益与预期收益或实际成本与预期成本 发生背离, 使其实际收益低于预期收 益, 或实际成本高于预期成本, 从而 使商业银行遭受损失的可能性。利率 风险可以为银行创造利润、提高股东 价值，但利率风险过度也会给银行带 来收益和资本的损失。

\section{1. 文献综述}

国外对商业银行利率风险管理的 研究比较早, 已经建立了比较完善的 利率风险管理体系。J.P.Morgan 公司

（1983）在其年报里将资产和负债按 期限分成多种类别，计算利率缺口的 数值, 第一次提出了利率敏感性缺口 的概念。Morgan and Smth (1997) 建 立了动态的利率敏感性缺口规划模 型。上世纪 90 年代, J.P.Morgan 公司 （1986）在风险管理实践中摸索出了 一种新的风险度量手段一 VaR 方法。

1994 年, G-30（1994）在《衍生产品 的实践和规则》中向各国金融机构推 荐了 VaR 方法。随后, Philippe Jorion

（1996）出版了第一部系统介绍 VaR 概念及应用的著作《风险价值》，该 书系统全面地讨论了 VaR 模型的数理 基础、计算过程与应用途径。

国内对商业银行的风险管理研究 起步较晚, 随着利率市场化的不断深 入, 国内开始重视对风险管理的研 究。牛昂, 郑文通 (1997) 介绍了 $\mathrm{VaR}$ 产生的背景、其基本概念和算 法。刘宇飞（1999）全面介绍了 $\mathrm{VaR}$, 指出了其出现对于金融监管的转 变具有重要意义。黄金老（2001）指 出利率进程化中利率风险分为阶段性 风险和恒久性风险。杨锦（2007）通
过对我国商业银行面临的利率风险的 现状进行分析, 得出导致利率风险的 主要因素使我国的制度性因素。

\section{2. 商业银行的利率风险分类}

巴塞尔银行监管委员会在《利率 风险管理与监管原则》中将银行利率 风险分为重新定价风险、基差风险、 收益曲线风险以及期权性风险等四 类, 见表 1 。

表 1：利率风险主要分类

\begin{tabular}{|c|c|}
\hline 风险种类 & 内容 \\
\hline $\begin{array}{l}\text { 重新定价风险 } \\
\text { ( Repricing } \\
\text { Risk) }\end{array}$ & $\begin{array}{l}\text { 也称为期限错配风险, 是最主 } \\
\text { 要和最常见的利率风险形式。 } \\
\text { 由银行固定利率下资产、负债 } \\
\text { 及表外业务的到期时间与浮动 } \\
\text { 利率下的重新定价期限不符而 } \\
\text { 导致。 }\end{array}$ \\
\hline $\begin{array}{l}\text { 基准风险（Basis } \\
\text { Risk） }\end{array}$ & $\begin{array}{l}\text { 也称为利率定价基础风险, 在 } \\
\text { 其他重新定价条件不变的条件 } \\
\text { 下, 银行的资产和负债以不同 } \\
\text { 的基准利率计息, 即利息的收 } \\
\text { 入和支出不完全匹配时, 利差 } \\
\text { 收入、现金流发生变动所引起 } \\
\text { 的利率风险。 }\end{array}$ \\
\hline $\begin{array}{l}\text { 收益曲线风险 } \\
\text { ( Yield Curve } \\
\text { Risk) }\end{array}$ & $\begin{array}{l}\text { 也称为利率期限结构变化风 } \\
\text { 险, 当由于重新定价的不对称 } \\
\text { 性使得收益曲线发生斜率及形 } \\
\text { 态上的变化时, 对银行的收益 } \\
\text { 或内在价值产生不利影响的可 } \\
\text { 能性。 }\end{array}$ \\
\hline $\begin{array}{l}\text { 期 权 性 风 险 } \\
\text { ( Optionality } \\
\text { Risk) }\end{array}$ & $\begin{array}{l}\text { 也称为选择权风险, 客户拥有 } \\
\text { 提前支取定期存款或提前偿还 } \\
\text { 贷款的选择权, 当利率发生变 } \\
\text { 动时, 银行因此蒙受损失的可 } \\
\text { 能性。 }\end{array}$ \\
\hline
\end{tabular}

\section{VaR 的基本原理及计算方法}

\subsection{VaR 的基本原理}

VaR（Value-at-Risks），即在险价 值，表示金融资产在各种风险下的暴 露程度。1996 年, 菲利普・乔瑞定义 $\mathrm{VaR}$ 为在正常市场情况下, 给定置信 区间内，某种投资工具或者资产组合 在一定的某一时期内的最大损失值。 用数学式表示如下: 


$$
\operatorname{Prob}(\Delta P>V a R)=1-\mathrm{c}
$$

在上式中 Prob 表示概率公式; $\Delta \mathrm{P}$ 表示某资产或资产组合在持有期内 的价值损失; $\mathrm{c}$ 表示选定的置信水平; $\mathrm{VaR}$ 表示置信水平 $\mathrm{c}$ 下的风险价值, 即可能达到的损失上限。

\subsection{VaR 模型的计算方法}

$\mathrm{VaR}$ 模型的计算通过根据模拟收 益率的不同形式来划分，而其估计的 结果取决于收益率的拟合的准确程 度。目前比较常用的计算 $\mathrm{VaR}$ 的方法 主要有：模拟参数收益率概率分布的 参数模拟法和不需要收益率概率分布 的非参数模拟法。本文研究使用参数 模拟法, 所以重点介绍参数法, 非参 数法不另做介绍。

参数法的核心是对资产价格或收 益的方差协方差矩阵的估计。代表是 方差一一协方差法。

方差一一协方差法假定了风险因 子或者金融资产是线性和正态的, 即 在持有期限内, 资产的价值变化与收 益率的关系呈线性，收益率服从正态 分布。对于现实中的金融资产时间序 列分布中的厚尾性及异方差性该假设 不能准确的描述, 但其操作简单的优 点使得其被广泛应用。

假设资产的初始价值为 $M_{0}$, 利 用金融工具进行投资, 回报序列为 $\left\{P_{\mathrm{t}}\right\}$, 每期收益率为 $R_{t}$, 令 $R_{\mathrm{t}}=\frac{P_{t}-P_{t-1}}{P_{t-1}}, R_{t}$ 的均值为 $\mu$, 方差 为 $\sigma^{2}$ 。给定置信水平 $\mathrm{c}$ 及标准正态分 布下相对应的取值 $\alpha$, 则可以求出 $\mathrm{VaR}$ 值，具体计算如下：

$$
\mathrm{c}=\int_{-\infty}^{-W} f(W) d W=\int_{-\infty}^{-R^{+}} f(r) d r=\int_{-\infty}^{-\alpha} \phi(\xi) d \xi
$$

其 中 $\frac{R^{*}-\mu}{\alpha}=-\alpha$, 则 $R^{*}=-\alpha \sigma+\mu$ 。于是,
$\operatorname{VaR}=-M_{0}\left(R^{*}-\mu\right)=M_{0} \alpha \sigma=Z_{\alpha} \sigma_{P} \sqrt{\Delta t}$

其中, $Z_{\alpha}$ 为值 $\alpha$ 对应的标准正态 分布分位数, $\sigma_{P}$ 为金融工具收益的标 准差, $\Delta t$ 为持有期限。

\section{3.我国商业银行利率风险度量实证研究}

\section{1 实证数据的选择及数据分析}

利率衡量的是单位货币在单位时 间内的价值。在利率市场化条件下， 利率的决定是通过资金供求双方达到 均衡状态时。1996 年我国建立了银行 间同业拆借市场，在我国银行间同业 拆借市场上，上海银行间同业拆放利 率是目前市场化水平最高的利率。目 前, 上海银行间同业拆放利率对外公 布的利率品种包括了隔夜, 1 周、2 周、 1 个月、 3 个月、 6 个月、 9 个月及 1 年期限等不同期限的 8 种拆借利率, 其中最为活跃利率品种为隔夜拆借利 率。因此，本文选取上海银行间同业 拆放利率中的隔夜拆借利率为样本, 应用 Eviews7 软件对样本进行数据分 析。

本文样本选取的时间跨度为 2010 年 1 月 4 日至 2016 年 12 月 30 日，样 本容量为 1748 个, 用于样本内模型估 计。数据来源于上海同业拆借利率网 站。在模型估计前, 选取置信水平为 $95 \%$, 持有期为 1 天来进行预测。

\section{(1) 平稳性检验。}

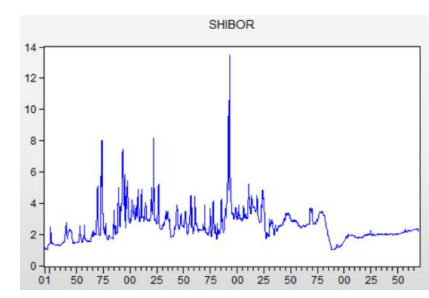

图 1: 隔夜拆借利率时间序列图

由图 1 可以发现其波动频繁, 数 值变动不平稳, 具有趋势性, 为了消 
除隔夜拆借利率的趋势性，对其取对 数收 益 率, 令 $\mathrm{r}_{t}=\log \left(\right.$ shibor $\left._{t}\right)-\log \left(\right.$ shibor $\left._{t-1}\right)$ 。

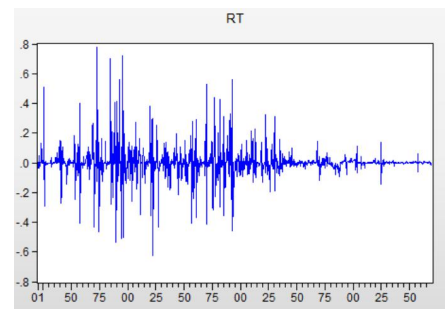

图 2: 对数收益率时间序列图

从图 2 可以看出, 收益率序列围 绕着一定的均值波动, 不存在明显的 趋势, 利用 $\mathrm{ADF}$ 平稳性检验, 证明其 平稳性, 结果如表 1 。可以看出, 该序 列的 $\mathrm{t}$ 值在 $1 \%$ 、 $5 \%$ 及 $10 \%$ 的置信水平 下，均明显小于 $\mathrm{t}$ 统计量的临界值，因 此拒绝单位根假设, 即认定序列是平 稳的。因此, 后续分析均基于对数收 益率序列展开。

表 2: ADF 检验结果

\begin{tabular}{|l|l|l|l|}
\hline $\begin{array}{l}\mathrm{ADF} \\
\text { 检验值 }\end{array}$ & $\begin{array}{l}1 \% \\
\text { 检验值 }\end{array}$ & $\begin{array}{l}5 \% \\
\text { 检验值 }\end{array}$ & $\begin{array}{l}10 \% \\
\text { 检验值 }\end{array}$ \\
\hline-37.75897 & -3.433892 & -2.862991 & -2.567590 \\
\hline
\end{tabular}

(2) 正态性检验。

若收益率服从正态分布, 则可以 直接由分位数求出相应置信区间下的 收益率, 因此, 先用 Q-Q 图法检验时 间序列是否具有正态性, 由图 3 可知 出现左下方向上弯曲, 右上方向下弯 曲的情况, 则说明数据存在厚尾现 象。

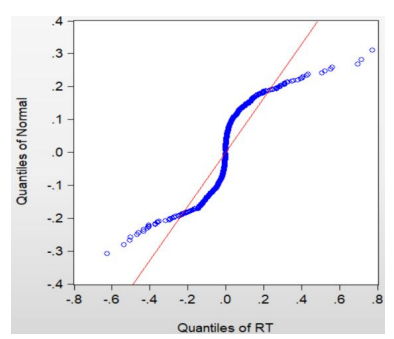

图 3：时间序列 $\mathrm{Q}-\mathrm{Q}$ 图
进一步, 采用直方图法对数据进 行处理, 如图 4:

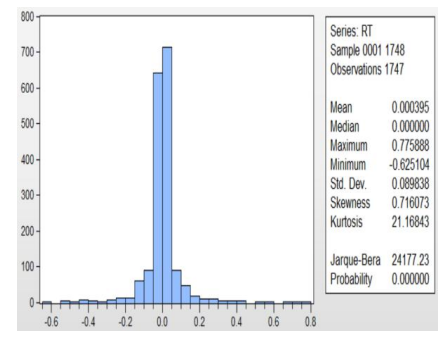

图 4: 时间序列直方图

由图 4 可知, 该序列的偏度为 0.72 , 峰度为 21.17 , Jarque-Bera 统计 量为 24177 , 均远大于正态分布的参数 估计。因此拒绝正态分布的原假设, 认定该序列并非正态分布, 存在尖峰 厚尾现象。

(3) 自相关检验。

\begin{tabular}{|c|c|c|c|c|c|}
\hline Autocorrelation & Partial Correlation & $A C$ & PAC & Q-Stat & Prob \\
\hline 中 & 卢 & 10.100 & 0.100 & 17.550 & 0.000 \\
\hline$\sqrt{1}$ & $\sqrt{1}$ & $2-0.037$ & -0.048 & 20.004 & 0.000 \\
\hline$\|$ & 中 & 30.013 & 0.022 & 20.320 & 0.000 \\
\hline 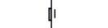 & d & $4-0.037$ & -0.043 & 22.745 & 0.000 \\
\hline 1 & di & $5-0.054$ & -0.045 & 27.871 & 0.000 \\
\hline II & i & $6-0.033$ & -0.027 & 29.807 & 0.000 \\
\hline 1 & 1 & $7-0.036$ & -0.033 & 32.087 & 0.000 \\
\hline di & पे & $8-0.087$ & -0.084 & 45.536 & 0.000 \\
\hline$[1$ & d & $9-0.079$ & -0.069 & 56.506 & 0.000 \\
\hline 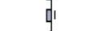 & 4 & $\begin{array}{ll}10 & -0.079\end{array}$ & -0.079 & 67.419 & 0.000 \\
\hline (1) & H & $11-0.010$ & -0.006 & 67.603 & 0.000 \\
\hline (1) & di & $12-0.018$ & -0.035 & 68.152 & 0.000 \\
\hline di & di & $13-0.035$ & -0.048 & 70.311 & 0.000 \\
\hline (1) & di & $14-0.020$ & -0.038 & 71.050 & 0.000 \\
\hline 中' & प' & $15-0.046$ & -0.067 & 74.797 & 0.000 \\
\hline
\end{tabular}

图 5: 时间序列相关图

由图 5 可以看出, 时间序列的各 阶自相关系数与偏自相关系数均不为 0 , 且在 $5 \%$ 的置信水平下, 可以拒绝 其不存在一阶自相关的原假设, 即可 以认定其存在序列自相关。

（4）条件异方差性检验。

先通过最小二乘法用 AR（1）模 型估计方程, 然后对残差序列进行一 阶滞后的 $\mathrm{ARCH}-\mathrm{LM}$ 检验, 得到表 2 , 发现其 $\mathrm{F}$ 统计量及 $R^{2}$ 统计量都是 显著的, 说明序列存在着 $\mathrm{ARCH}$ 效 应。 
表 3: 时间序列的 ARCH-LM 检验

\begin{tabular}{|l|c|l|c|}
\hline $\begin{array}{l}\text { F- } \\
\text { statistic }\end{array}$ & 120.4847 & $\begin{array}{l}\text { Prob. F (1,1 } \\
743)\end{array}$ & 0.0000 \\
\hline $\begin{array}{l}\text { Obs*R- } \\
\text { squared }\end{array}$ & 112.8240 & $\begin{array}{l}\text { Prob. Chi- } \\
\text { Square (1) }\end{array}$ & 0.0000 \\
\hline
\end{tabular}

是否存在 $\mathrm{ARCH}$ 效应也可以应用 残差平方的自相关图来验证。

\begin{tabular}{|c|c|c|c|c|c|c|}
\hline Autocorrelation & Partial Correlation & & $A C$ & PAC & Q-Stat & Prob \\
\hline 扫 & 曰 & 1 & 0.254 & 0.254 & 113.08 & 0.000 \\
\hline 舟 & 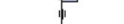 & 2 & 0.080 & 0.016 & 124.27 & 0.000 \\
\hline (19 & is & 3 & 0.100 & 0.081 & 141.88 & 0.000 \\
\hline in & 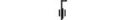 & 4 & 0.054 & 0.009 & 14701 & 0.000 \\
\hline 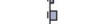 & 6 & 5 & 0.111 & 0.096 & 168.55 & 0.000 \\
\hline 傿 & 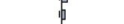 & 6 & 0.107 & 0.054 & 188.77 & 0.000 \\
\hline 巾 & in & 7 & 0.063 & 0.016 & 195.65 & 0.000 \\
\hline 舟 & 1) & 8 & 0.080 & 0.046 & 207.00 & 0.000 \\
\hline 占 & . & 9 & 0.086 & 0.044 & 220.04 & 0.000 \\
\hline 1 & 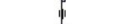 & 10 & 0.068 & 0.022 & 228.12 & 0.000 \\
\hline 紧 & b & 11 & 0.105 & 0.066 & 247.66 & 0.000 \\
\hline$\sqrt{5}$ & i & 12 & 0.071 & 0.012 & 256.63 & 0.000 \\
\hline in & i & 13 & 0.052 & 0.012 & 261.39 & 0.000 \\
\hline "i & di & 14 & 0.019 & -0.029 & 262.00 & 0.000 \\
\hline 巾 & 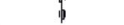 & 15 & 0.045 & 0.027 & 265.62 & 0.000 \\
\hline
\end{tabular}

图 6: 残差平方相关图

由图 6 可以看出, 收益率序列是 存在条件异方差性的, 其各阶的自相 关 (AC)、偏自相关 (PAC) 系数均 不为 0 , 且 $\mathrm{Q}$ 统计量均是显著的, 表 明了残差平方序列具有较强的相关 性。

\section{2 建立 GARCH 模型求得 VaR 值}

本文使用 $\operatorname{GARCH}(1,1)$ 模型对利率 的波动性进行分析, 建立条件均值方 程为 $r_{t}=c+c(1) r_{t-1}+u_{t}$, 结果如 下:

\begin{tabular}{ccccc}
\hline \hline Variable & Coefficient & Std. Error & z-Statistic & Prob. \\
\hline \hline C & 0.000840 & 0.001330 & 0.631990 & 0.5274 \\
RT(-1) & 0.272427 & 0.025583 & 10.64860 & 0.0000 \\
\hline \hline \multicolumn{5}{c}{ Variance Equation } \\
\hline C & 0.000289 & $7.03 E-06$ & 41.10554 & 0.0000 \\
RESID(-1)2 & 0.367513 & 0.018346 & 20.03257 & 0.0000 \\
GARCH(-1) & 0.739796 & 0.006677 & 110.8037 & 0.0000 \\
\hline \hline R-squared & -0.019690 & Mean dependentvar & 0.000397 \\
Adjusted R-squared & -0.020275 & S.D. dependent var & 0.089864 \\
S.E. of regression & 0.090771 & Akaike info criterion & -2.620502 \\
Sum squared resid & 14.36931 & Schwarz criterion & -2.604852 \\
Log likelihood & 2292.698 & Hannan-Quinn criter. & -2.614716 \\
Durbin-Watson stat & 2.303258 & & & \\
\hline \hline
\end{tabular}

图 7: GARCH $(1,1)$ 估计结果

图 7 为估计的 $\operatorname{GARCH}(1,1)$ 模 型, 估计结果为:
条件均 值方 程: $r_{t}=0.0008+0.2724 r_{t-1}+u_{t}$

条件 方 差 方 程 : $\sigma_{\mathrm{t}}^{2}=0.0003+0.3675 u_{t-1}^{2}+0.7398 \sigma_{\mathrm{t}-1}^{2}+\varepsilon_{t}$ 由表 3 可以看出, 均值方程中滞后项 的相伴概率为 0 。残差、 $\mathrm{ARCH}$ 项、 GARCH 项的相伴概率均为 0 , 低于 0.05 的置信水平, 这可以看出方差方 程是显著的, 模型能够较好地拟合数 据。对方差方程进行条件异方差检 验, 取滞后阶数为 2 , 得到 $\mathrm{F}$ 统计量的 伴随概率为 0.9680 , 可以认为模型已 经充分消除了条件异方差效应。

$$
\text { 由 } V a R=-M_{0}\left(R^{*}-\mu\right)=M_{0} \alpha \sigma=Z_{\alpha} \sigma_{P} \sqrt{\Delta t} \text {, }
$$

以数据的经验分布估计分位数 $\hat{Z_{0.005}}=1.644854$, 由模型估 计 $\hat{\sigma}_{\mathrm{t}}=1.1076$, 假设银行每日投放在同 业拆借市场的资金为 $10^{7}$ 元人民币， 则 根 据 可 得 $R_{\mathrm{i}}=\frac{r_{i}}{365}$, 可 的 $\hat{\sigma}_{P}=3034.54$, 令持有期 $\Delta \mathrm{t}=1$, 则 可以计算出 $\mathrm{VaR}$ ，即银行投放于同业 拆借市场的一千万元人民币，在 $95 \%$ 的置信水平下由于利率波动所导致的 损失不超过 10 万元。

\section{4.小结}

（1）我国的银行同业拆借市场跟 股票市场类似，都存在波动聚集性， 具有尖峰厚尾的特征。同业拆借市场 的收益率受到前一天的影响, 即存在 自相关性。

(2) GARCH 模型存在对条件分 布的假设不正确的问题，所以没有完 全解决同业拆借收益率序列中 “尖峰 厚尾” 的问题, 这种情况主要是由于 $\varepsilon_{\mathrm{t}}$ 的无条件分布存在厚尾现象, 而且 在大多数情况下 $\varepsilon_{\mathrm{t}}$ 的条件分布也是非 
正态的。利用 VaR 模型进行正态分布 假设计算时, 收益率分布厚尾现象越 突出, 其数值的估计偏差越大, 对商 业银行的风险管理会产生影响。

\section{5.结论与展望}

运用 VaR 方法测度利率风险可以 在一定程度上提高我国金融市场整体 的监管水平。监管部门可以以风险评 估作为基础, 选择适合的风险水平, 利用 VaR 方法对市场风险进行有效监 管。对于银行业来说, 加强 $\mathrm{VaR}$ 模型 应用于利率风险管理, 对商业银行利 率风险的管理具有重要意义。

\section{1 数据信息系统创建}

$\mathrm{VaR}$ 模型的运用需要大量的数据来 作数据支撑，数据的缺乏容易造成不 准确的参数估计, 进而影响估计得损 失值。如果想利用 VaR 方法进行利率 风险管理, 就需要大量数据作建模基 础。为了更好的做好风险控制, 商业 银行要积极主动的积累利率变动的数 据, 能够利用历史模拟与方差一协方 差法两种方法相互配合, 发挥其各自 优势, 进而度量利率风险。

\section{2 置信水平、持有期长度的选择要 量身定做}

我国商业银行在利用 $\mathrm{VaR}$ 模型 时, 会有一些不利因素, 实际能力的 制约和数据的不足。结合现有实际情 况, 持有长度选取 1 个交易日, 置信 水平选择 $95 \%$, 是目前较好的选择。 但是，随着我国进行金融体制的改 革、经济结构调整, 金融市场变幻莫 测，其他利率、汇率、金融资产价格 波动等可能带来的风险, 商业银行也 应该加以度量和预防。

\section{参考文献}

[1] Morgan J.P. RiskMetrics-Technical Document[M].New York:Morgan Guaranty Trust Co,pany Global Research.1996

[2] Jakobsen S.Measuring value-at-risk for mortgage backed securities[M].Springer US.1996.

[3] Jorion P.How informative are valueat-risk disclosures?[J].The Accounting Review,2002,77(4):911931.

[4] 巴塞尔银行监管委员会.利率风险管 理与监管原则.来源: 银监会官方网 站, 2004,7.

[5] 高铁梅.计量经济分析方法与建模: Eviews 应用及实例（第二版） [M]. 清华大学出版社.2009.

[6] 黄金老.利率市场化与商业银行风险 控制 [J].经济研究.2001(1):19-28.

[7] 武剑.利率市场化进程中的利率风险 管理 [J].经济研究.2003(2):58-63.

[8] 朱霞, 刘松林.利率市场化背景下商 业银行利率风险管理 $[\mathrm{J}]$. 金融理论 与实践, 2010 年第 2 期.

[9] 张宁.VaR 在我国商业银行利率风险 衡量中的应用分析[D].西南财经大 学.

[10] 张慧琳. 基于 $\mathrm{ARCH}$ 模型族的 $\mathrm{VaR}$ 方法在商业银行利率风险管 理中的应用[D].山东大学. 